\title{
The Effect of Whole-Body Vibration Warm-Up on Performance of Wingate Anaerobic Test
}

\author{
Morteza Afsari ${ }^{1, *}$; Mohammad Reza Ramezanpour ${ }^{2}$; Seyed Mahmoud Hejazi ${ }^{2}$; Mahdi \\ Bayati $^{3}$; Babak Farzad ${ }^{4}$ \\ ${ }_{2}^{1}$ Khorasan Razavi Education Organization, Mashhad, IR Iran \\ ${ }^{2}$ Department of Physical Education and Sports Sciences, Mashhad Branch, Islamic Azad University, Mashhad, IR Iran \\ ${ }^{3}$ Department of Physical Education and Sports Sciences, Faculty of Humanities, Tarbiat Modares University, Tehran, IR Iran \\ ${ }^{4}$ Department of Exercise Physiology, Faculty of Physical Education and Sports Sciences, Kharazmi University, Tehran, IR Iran \\ *Corresponding author: Morteza Afsari, Khorasan Razavi Education Organization, Mashhad, IR Iran. E-mail: afsari.morteza@gmail.com
}

Received: May 16, 2013; Revised: August 6, 2013; Accepted: January 2, 2014

\section{Dear Editor,}

Whole-body vibration training (WBV) has been considered as a novel training method that causes mechanical stimulation in neuromuscular system. Recently, investigators suggested that mechanical stimulation with low frequencies and appropriate amplitudes is an effective way to prepare muscular structures [1]. Therefore, in the present study, we tried to have new approach to this training method and studied the effects of two warm-ups, traditional and WBV warm-up, on performance of Wingate test (e.g. a standard test to evaluate anaerobic power and capacity). Ten physically active male college students volunteered to participate in the study (age $=25 \pm 0.8$ years; height $=1.72 \pm 0.08 \mathrm{~m}$; mass $=70 \pm 11 \mathrm{~kg}$; percent body fat $=$ $18 \pm 6 \%$ ). The subjects completed the medical assessment questionnaire. Prior to any participation, the experimental procedures and potential risks were explained fully to the participants and all provided written informed consent. All participants attended a laboratory familiarization visit to introduce the testing/training procedures before baseline testing. These crossover trials were conducted in two days separated by 3 days. At the first day, Subjects used traditional warm-up and three days later, they warmed up with usage of WBV [10 bouts of $30 \mathrm{sec}-$ onds vibration and 30 seconds rest, frequency was set to $26 \mathrm{~Hz}$, amplitude of the vibration platform was $10 \mathrm{~mm}$ in half squat position (flexion $100^{\circ}$ )] [2]. After every warmup session, subjects performed a 30 second all-out Wingate test. All data were analyzed using dependent t-tests with SPSS-16. The alpha level for statistical significance was set at $\mathrm{P} \leq 0.05$. There was a significant difference between two methods of warm-up in just variable of mini- mum power output, and no differences between two methods of warm-up in peak power output, mean power output, and fatigue index were not observed. WBV warmup causes stimulation and recruitment of the most motor units, and also increases in muscle temperature and muscle blood flow which is due to the augmentation of metabolism in major muscular groups $[1,3]$; consequently, these events cause improvement in anaerobic power which can explained the improvement of minimum power output by this mechanism.

The most important finding of the present study was that we did not observe any differences between two methods of warm-up (whole-body vibration and traditional warm-up) on anaerobic performance of the active males and that these results possibly refer to intensity, duration, and amplitude of the vibration, because changes in each of them can have different effects on force and power output. Since there is no consensus on the presentation of a specific protocol with certain frequency, amplitude and duration of WBV in warm-up period; therefore, more investigations in this field are required.

\section{References}

1. Delecluse C, Roelants M, Verschueren S. Strength increase after whole-body vibration compared with resistance training. Med Sci Sports Exerc. 2003;35(6):1033-41.

2. Bosco C, Cardinale M, Tsarpela O. Influence of vibration on mechanical power and electromyogram activity in human arm flexor muscles. Eur J Appl Physiol Occup Physiol.1999;79(4):306-11.

3. Cochrane DJ, Stannard SR, Sargeant AJ, Rittweger J. The rate of muscle temperature increase during acute whole-body vibration exercise. EurJ Appl Physiol. 2008;103(4):441-8. 